\title{
Using a cold test to assess pulpal anaesthesia
}

\author{
Is a cold test effective in determining whether a patient will experience pain \\ during root canal therapy?
}

\section{Hsiao-Wu GW, Susarla SM, White RR.}

Use of the cold test as a measure of pulpal anesthesia during endodontic therapy: a randomized, blinded, placebo-controlled clinical trial. J Endod 2007; 33:406-410

Design This was a randomised controlled trial (RCT).

Intervention Groups given a true cold test (test group) or a sham cold test (control) were compared.

Outcome measure If pain was experienced during the procedure, patients were asked to point to their level of pain on a visual analogue scale (VAS). Stages of the RCT were divided as follows: before entering the pulp chamber, while entering the pulp chamber, preparing the canal, irrigating the canals, and obturation of the canals.

Results Unadjusted results showed $12 \%$ of test subjects experienced pain during the RCT compared with $38 \%$ of control subjects $(\mathrm{N}=83$; $P$ 0.004; power, 84\%). Multiple logistic regression analysis controlled for confounders and effect-modifiers (odds ratio, $0.20 ; P 0.01$ ). Subjects who had a negative response to the cold test were approximately $80 \%$ less likely to experience pain during the procedure than subjects who had only soft tissue signs of anaesthesia.

Conclusions The cold test is a significantly better indicator of pulpal anaesthesia than the current standard of care, ie, using soft tissue signs alone. We strongly advocate the use of the cold test to assess pulpal anaesthesia.

\section{Commentary}

An assessment of adequate pulpal anaesthesia is not as easy a task as it may seem. A critical review of the literature produces the question, "Is there any single test that we can rely upon with full confidence?" Unfortunately, the answer has always been, "No." ${ }^{1}$ Traditional methods of confirming anaesthesia are usually subjective and involve verbal questioning of the patient about his/ her symptoms, or soft-tissue testing, where the response is noted to a sharp explorer. These approaches may not be effective for determining pulpal anaesthesia and provide only bimodal responses; hence they cannot detect parametric differences between anaesthetics or routes of injection. Alternatively, anaesthesia can be measured more objectively by a variety of sensitivity tests, using either cold, heat or an electrical stimulus.

There is growing recognition that evidence-based therapies are a valuable addition to the treatment options to be considered along with the dental practitioner's skills and the patient's needs. In many areas of dentistry, there are limited numbers because few randomised, placebo-controlled double-blind clinical trials have been conducted.

Address for correspondence: Dr Grace W Hsiao-Wu, Division of Endodontics, Harvard School of Dental Medicine, 188 Longwood Avenue, Boston MA 02115, USA. E-mail: grace_hsiao@post.harvard.edu
The current report is such a trial, and represents an interesting addition to the literature. The design, methods and statistical analysis show convincingly that the traditional methods of assessment are not efficient, and that another test is required to confirm adequate pulpal anaesthesia. The analysis of associations between predictor variables and reported pain is very interesting and seems to be in agreement with previous reports in the fields.

Although in this model the authors did not look into efficiency of pulpal anaesthesia in relation to the anaesthetic technique, the type and volume of anaesthetic did not seem to have an effect. For many practitioners, administering an intraligamentary injection following regional ones is a day-to-day practice. ${ }^{2}$ Intraosseous injections, however, have been shown to be the most effective in controlling pain during nonsurgical root canal treatment, particularly during access opening. ${ }^{3}$

What about the electrical pulp testers or hot tests? Might they be better predictors than cold tests, particularly for teeth not responsive to cold at baseline? Testing adjacent teeth, as predictors for pain, might lead to false negatives because the immunopharmacological environment of the pulps and the periradicular areas are different from the pathologically involved ones. ${ }^{4}$

\section{Practice points}

The use of a cold test after obtaining subjective symptoms of anaesthesia is a reliable way to assess the efficiency of profound pulpal anaesthesia, particularly if pain to cold was noted at the diagnosis stage.

Practitioners should be familiar with the various approaches for endodontic anaesthesia, particularly intra-osseous ones, to minimise anaesthetic failure.

Anticipating patients who might be resistant to anaesthesia utilising the predictors mentioned in this and other studies could help in overall management.

\section{Khaled Balto}

Faculty of Dentistry, King Abdulaziz University, Jeddah, Saudi Arabia

1. McLean C, Reader A, Beck M, Meyers WJ. An evaluation of $4 \%$ prilocaine and $3 \%$ mepivacaine compared with $2 \%$ lidocaine (1:100,000 epinephrine) for inferior alveolar nerve block. J Endod 1993; 19:146-150.

2. Childers M, Reader A, Nist R, Beck M, Meyers W]. Anaesthetic efficacy of the periodontal ligament injection after an inferior alveolar nerve block. J Endod 1996; 22:317-320.

3. Coggins R, Reader A, Nist R, Beck M, Meyers WJ. Anaesthetic efficacy of the intraosseous injection in maxillary and mandibular teeth. Oral Surg Oral Med Oral Pathol Oral Radiol Endod 1996; 81:634-641.

4. Stashenko P, Teles R, D'Souza R. Periapical inflammatory responses and their modulation. Crit Rev Oral Biol Med 1998; 9:498-521.

Evidence-Based Dentistry (2007) 8, 102. doi:10.1038/sj.ebd.6400522 